\title{
Sulle coordinate curvilinee d' una superficie e dello spazio.
}

\author{
MEMORIA QUARTA (*). \\ (del prof. Delfino Codazzi, a Pavia).
}

\begin{abstract}
I signor prof. Aoust ha già trattato in un modo bastantemente esteso il problema generale delle coordinate curvilinee dello spazio, risguardate come i parametri di tre sistemi di superficie segantisi ad angolo qualunque, ed ha già presentato l'applicazione della sua soluzione ad una linea tracciata in una superficie. Si veda, per la trattazione generale, la Théorie des coordonnées curvilignes quelconques inserita negli Annali di matematica pura ed applicata pubblicati a Roma nell'anno 1864 (serie $1^{2}$, t. 6), del qual lavoro io non aveva cognizione quando stampava la mia Memoria prima. Il metodo perơ da lui tenuto differisce essenzialmente da quello puramente analitico seguito dal signor LaMÉ nel trattare il problema delle coordinate curvilinee ortogonali, e quindi anche da quello seguito da me nel trattare il problema delle coordinate curvilinee qualunque. Il signor Aoust osserva su questo punto (V. Annali, s. $1^{\mathrm{a}}$, t. $6^{\circ}$, p. 65): «Pour obtenir la solution de la question a point de vue le plus général, il nous a été impossible de profiter de la méthode suivie par Mr. Lamé dans le cas des coordonnées orthogonales à cause des complications qui s'attachent à la question générale. En effet, les trois cosinus des angles coordonnés, leurs variations premières et secondes par rapport aux trois paramètres qui fixent la position du point, s'introduisent nécessairement dans les équatious, et lorsque l'on suit la méthode analytique, ces quantités au nombre de 30, qui n'existent pas dans le système orthogonal,
\end{abstract}

(*) I numeri adoperati per indicare le formole di questa Memoria quarta sono in continuazione con quelli della Memoria terza. 
entrent dans la composition des coefficients d'un systeme de neuf équations linéaires à neuf inconnues a uquel cette méthode conduit juévitablement. Or, comme la résolution de ce système d'équations est indispensable, les difficultés analytiques de cette résolution imposent la nécessité de renoncer à la marche tracée par l'auteur de la théorie des coordonnées orthogonales.» Il metodo seguíto dal signor Aoust è geometrico piuttosto che analitico, e poggia principalmente sulla considerazione di un elemento, la curvatura inclinata, nuovamente introdotto da esso nella teoria. «La simplification, egli continua, due à la marche qui nous est propre, résulte de son caractère essentiellement géométrique, et surtout d'un élément géométrique nouveau que nous avons introduit dans notre théorie. Nous appelons cet élément courbure inclinée des lignes coordonnées. Il ne sert pas seulement à éviter des calculs impraticables, mais il est un instrument précieux de transformation et de démonstration, et son introduction dans les équations leur donne une forme à la fois simple et significative. »I risultati, ai quali giunge il signor Aoust nel suo lavoro sul problema generale (Ann., s. $1^{\text {a }}$, t. $6^{\circ}$ ) consistono in diverse equazioni stabilite fra le seguenti 33 quantità: le 9 componenti oblique della curvatura ordinaria, le 18 componenti oblique della curvatura inclinata, e le 6 quantità da me chiamate $l, m, n, \varepsilon_{\eta}, \varepsilon_{\mu}, \varepsilon_{\nu}$. Alcune fra queste equazioni sono finite, ed altre sono alle derivate parziali del prim'ordine rispetto a' tre parametri (*).

(*) Anche il signor professor Domenico Chelini, in parte d'una sua Memoria inserita negli Annali di Tortolini del 1853, della quale pure io non aveva cognizione quando scriveva la mia Memoria prima, ha preso a considerare le coordinate curvilinee dello spazio come i parametri di tre sistemi di superficie segantisi ad angolo qualunque. Egli ha somministrato una trasformazione della quantità rappresentabile mediante le mie notazioni con $\Sigma \frac{\partial^{2} f}{\partial i^{2}}$, ove $f$ è funzione qualunque delle $\lambda, \mu, \nu$, ed ha costituito qualche formola relativa alla curvatura delle superficie. In quanto ad una noterella inserita dallo stesso signor prof. Chelini al piè della pagina 30 della sua recente Memoria: Della curvatura delle superficie con metodo diretto ed intuitivo, stampata a Bologna nel 1868 , amerei far notare che $\mathrm{i}$ miei risultati consistono in certe 9 espressioni presentate in funzioni delle $l, m, n, \varepsilon_{\lambda,} \varepsilon_{\mu}, \varepsilon_{y}$ e loro derivate, in funzioni cioe di novelle quantità di numero possibilmente minimo e di significazione geometrica evidente; e che, dopo d'avere costituito i valori di quelle 9 espressioni, ho anche implicitamente costituito il valore generale della $\Sigma \frac{\partial^{2} f}{\partial \imath^{2}}$, ove $f$ è funzione qualunque delle $\lambda, \mu, \nu$, in causa di una formula di calcolo differenziale. 
Siccome le componenti oblique della curvatura ordinaria delle tre linee coordinate $(\mu, \nu),(\nu, \lambda),(\lambda, \mu)$, e quelle della curvatura inclinata nuovamente introdotta, possono essere adoperate come atile mezzo di ricerca in alcune questioni, cosi mi propongo di trattenermi sopra di esse nella presente Memoria quarta. Propriamente, trovero dapprima i valori di queste 27 componenti oblique espresse in funzioni delle $l, m, n, \varepsilon_{\lambda}, \varepsilon_{\mu}, \varepsilon_{\nu}$ e loro derivate parziali prime; perocchè, onde ultimare le integrazioni nei casi particolari, io credo necessario il possedere queste espressioni analitiche. Presenterò di più le stesse ventisette equazioni sotto altra forma; cioè troveró le equazioni finite, in numero di dodici, che legano tra loro le componenti oblique e le $l, m, n, \varepsilon_{\eta}, \varepsilon_{\mu}, \varepsilon_{\nu}$; e troverò le equazioni, in numero di quindici, che servono ad esprimere le derivate prime parziali delle $l, m, n$, $\varepsilon_{\lambda}, \varepsilon_{\mu}, \varepsilon_{\nu}$ in funzioni di queste sei quantità e delle componenti oblique. Parecchie tra le ventisette equazioni di questa seconda forma sono già state costituite con altro metodo dal signor Aoust.

1.

Cominciamo dalk'esprimere le nove componenti oblique della curvatura delle tre linee coordinate in funzioni delle $l, m, n, \varepsilon_{\mu}, \varepsilon_{\mu}, \varepsilon_{\nu}$ e loro derivate prime.

La curvatura della linea coordinata $(\mu, \nu)$, cioè l'unità divisa pel raggio di curvatura di questa linea, ha le tre projezioni sopra i tre assi rettilinei rappresentate da $\frac{1}{l} \frac{\partial i_{2}}{\partial \lambda}$. Chiamiamo $\frac{1}{L_{\lambda}}, \frac{1}{M_{\lambda}}, \frac{1}{N_{\lambda}}$ le tre compononenti oblique di questa curvatura secondo le tangenti alle tre linee coordinate $(\mu, v)$, $(\nu, \lambda),(\lambda, \mu)$, cioè le tre componenti formate dietro la regola del parallelepipedo delle forze; ed avremo, per nota proprietà,

$$
\frac{1}{l} \frac{\partial i_{2}}{\partial \lambda}=\frac{i_{2}}{L_{\lambda}}+\frac{i_{\mu}}{M_{\lambda}}+\frac{i_{\nu}}{N_{\lambda}}
$$

Questa formola, moltiplicata successivamente per $I_{\lambda}, I_{\mu}, I_{\nu}$ e sottoposta ciascuna volta all'operazione $\boldsymbol{\Sigma}$, somministra

$$
\frac{\boldsymbol{\Sigma} I_{2} i_{2}}{L_{\lambda}}=\frac{\boldsymbol{\Sigma} I_{\lambda} \frac{\partial i_{2}}{\partial \lambda}}{l}, \quad \frac{\boldsymbol{\Sigma} I_{\mu} i_{\mu}}{M_{\lambda}}=\frac{\boldsymbol{\Sigma} I_{\mu} \frac{\partial i_{2}}{\partial \lambda}}{l}, \quad \frac{\boldsymbol{\Sigma} I_{\nu} i_{\nu}}{N_{\lambda}}=\frac{\boldsymbol{\Sigma} I_{\nu} \frac{\partial i_{\lambda}}{\partial \lambda}}{l} .
$$


Ora, dalla prima (35) si deduce

$$
\begin{aligned}
\Sigma I_{2} \frac{\partial i_{2}}{\partial \lambda}=l^{\prime} \boldsymbol{\Sigma} I_{\lambda} i_{\lambda}^{\prime} & =l_{\{}^{\prime}\left\{\cos \omega_{\mu}^{\prime}\left(\cos \omega_{\mu} \cos \omega_{\lambda}^{\prime}+\operatorname{sen} \omega_{\mu} \operatorname{sen} \omega_{\lambda}^{\prime} \cos \varepsilon_{\nu}\right)\right. \\
& \left.+\operatorname{sen} \omega_{\mu}^{\prime}\left(\operatorname{sen} \omega_{\mu} \cos \omega_{2}^{\prime}-\cos \omega_{\mu} \operatorname{sen} \omega_{2}^{\prime} \cos \varepsilon_{\nu}\right)\right\}
\end{aligned}
$$

ovvero

$$
\Sigma I_{\lambda} \frac{\partial i_{2}}{\partial \lambda}=-\alpha_{2} \cos \eta_{\mu}-\beta_{\lambda} \operatorname{sen} \eta_{\mu} \cos \varepsilon_{\nu}
$$

e dalle (29) si deducono

$$
\Sigma I_{\mu} \frac{\partial i_{2}}{\partial \lambda}=l^{\prime} \Sigma I_{\mu} i_{\lambda}^{\prime}=a_{2}, \quad \Sigma I_{\nu} \frac{\partial i_{\nu}}{\partial \lambda}=l^{\prime} \mathbf{\Sigma} I_{\nu} i_{\lambda}^{\prime}=a_{\lambda}
$$

Percio saranno, in causa anche delle (24),

$$
\begin{aligned}
& \frac{l \bigvee \Delta}{L_{\lambda}}=-\operatorname{sen} \varepsilon_{\lambda}\left(\alpha_{\lambda} \cos \eta_{\mu}+\beta_{\lambda} \operatorname{sen} \eta_{\mu} \cos \varepsilon_{\nu}\right), \\
& \frac{l \bigvee \Delta}{M_{\lambda}}=a, \operatorname{sen} \varepsilon_{\mu}, \\
& \frac{l \bigvee \Delta}{N_{\lambda}}=\alpha_{\lambda} \operatorname{sen} \varepsilon_{\nu} .
\end{aligned}
$$

Le (77) esprimono le tre componenti oblique della curvatura della linea coordinata $(\mu, \nu)$ nel modo dichiarato, purchè s'intendano sostituiti in luogo delle $\alpha_{h}, \alpha_{\lambda}, \beta_{\lambda}$ i valori dati dalle (62), (63), (64). Mediante le permutazioni semplice e doppia si concluderanno dalle (77) dapprima le tre componenti oblique per la linea $(\nu, \lambda)$, dipoi le tre per la $(\lambda, \mu)$, purchè si cambino le $L, M, N$ nelle $M, N, L$ nel primo caso e nelle $N, L, M$ nel secondo.

Se le superficie de' tre sistemi fossero ortogonali tra loro, le (77) si ridurrebbero alle

$$
\frac{1}{L_{j}}=0, \frac{1}{M_{2}}=-\frac{1}{\operatorname{lm}} \frac{\partial l}{\partial \mu}, \frac{1}{N_{2}}=-\frac{1}{\ln } \frac{\partial l}{\partial \nu} .
$$

2.

Avanti di passare alle componenti oblique della curvatura inclinata della linea $(\mu, \nu)$, preparo i valori delle seguenti sei espressioni:

$$
\mathbf{\Sigma} I_{\lambda} \frac{\partial i_{\lambda}}{\partial \mu}, \quad \mathbf{\Sigma} I_{\mu} \frac{\partial i_{\lambda}}{\partial \mu}, \quad \mathbf{\Sigma} I_{\nu} \frac{\partial i_{\gamma}}{\partial \mu}, \quad \mathbf{\Sigma} I_{\lambda} \frac{\partial i_{\lambda}}{\partial \nu}, \quad \mathbf{Z} I_{\mu} \frac{\partial i_{\lambda}}{\partial \nu}, \quad \mathbf{\Sigma} I_{\nu} \frac{\partial i_{\lambda}}{\partial \nu}
$$


Le due (39) somministrano

$$
\begin{aligned}
& \Sigma I_{\mu} \frac{\partial i_{\mu}}{\partial \nu}=\cos \omega^{\prime}{ }_{\nu}\left(-\frac{\partial \operatorname{sen} \varepsilon_{\lambda}}{\partial \nu} \operatorname{sen} \omega_{\nu}+n^{\prime} \cos \varepsilon_{\lambda}+c_{\nu} \operatorname{sen} \varepsilon_{\lambda} \cos \omega_{\nu}\right) \\
& +\operatorname{sen} \omega_{\nu}^{\prime}\left(\frac{\partial \operatorname{sen} \varepsilon_{\lambda}}{\partial \nu} \cos \omega_{\nu}+c_{\nu} \operatorname{sen} \varepsilon_{i} \operatorname{sen} \omega_{\nu}\right), \\
& \sum I_{\nu} \frac{\partial i_{\nu}}{\partial \mu}=\cos \omega_{\mu}\left(\frac{\partial \operatorname{sen} \varepsilon_{i}}{\partial \mu} \operatorname{sen} \omega^{\prime} \mu+m^{\prime} \cos \varepsilon_{,}-\gamma_{\mu} \operatorname{sen} \varepsilon_{\gamma} \cos \omega_{\mu}^{\prime}\right) \\
& -\operatorname{sen} \omega_{\mu}\left(\frac{\partial \operatorname{sen} \varepsilon_{2}}{\partial \mu} \cos \omega^{\prime} \mu+\gamma \mu \operatorname{sen} \varepsilon_{2} \operatorname{sen} \omega^{\prime} \mu\right)
\end{aligned}
$$

ovvero

$$
\begin{aligned}
& \mathbf{\Sigma} I_{\mu} \frac{\partial i_{\mu}}{\partial \nu}=\operatorname{sen} \eta_{\nu} \frac{\partial \operatorname{sen} \varepsilon_{\lambda}}{\partial \nu}+a_{v} \cos \varepsilon_{\lambda}-c_{\nu} \cos \eta_{\nu} \operatorname{sen} \varepsilon_{\lambda_{i}} \\
& \mathbf{\Sigma} I_{\nu} \frac{\partial i_{\nu}}{\partial \mu}=\operatorname{sen} \eta_{\mu} \frac{\partial \operatorname{sen} \varepsilon_{\lambda}}{\partial \mu}+a_{\mu} \cos \varepsilon_{\lambda}+\gamma_{\mu} \cos \eta_{\mu} \operatorname{sen} \varepsilon_{\lambda}
\end{aligned}
$$

o anche

$$
\begin{aligned}
& \sum I_{\mu} \frac{\partial i_{\mu}}{\partial \nu}=-\operatorname{sen} \eta_{\nu} \cos \varepsilon_{\lambda}\left(b_{\nu}-\frac{\partial \varepsilon_{\lambda}}{\partial \nu}\right)-\cos \eta_{\nu}\left(a_{\nu} \cos \varepsilon_{\lambda}+c_{\nu} \operatorname{sen} \varepsilon_{2}\right), \\
& \sum I_{\nu} \frac{\partial i_{\nu}}{\partial \mu}=\operatorname{sen} \eta_{\mu} \cos \varepsilon_{2}\left(\beta_{\mu}+\frac{\partial \varepsilon_{\lambda}}{\partial \mu}\right)-\cos \eta_{\mu}\left(\alpha_{\mu} \cos \varepsilon_{\lambda}-\gamma_{\mu} \operatorname{sen} \varepsilon_{2}\right) ;
\end{aligned}
$$

perocchè sono, in causa delle $(6 \dot{2}),(63)$,

$$
\begin{gathered}
\alpha_{\nu}=-b_{\nu} \operatorname{sen} \eta_{\nu}-a_{\nu} \cos \eta_{\nu}, \\
a_{\mu}=\beta_{\mu} \operatorname{sen} \eta_{\mu}-\alpha_{\mu} \cos \eta_{\mu} .
\end{gathered}
$$

Operando la permutazione doppia sulla prima formola e la permutazione semplice sulla seconda, concludiamo

$$
\left.\begin{array}{l}
\mathbf{\Sigma} I_{\lambda} \frac{\partial i_{\lambda}}{\partial \mu}=-\operatorname{sen} \eta_{\mu} \cos \varepsilon_{\nu}\left(b_{\mu}-\frac{\partial \varepsilon_{\nu}}{\partial \mu}\right)-\cos \eta_{\mu}\left(a_{\mu} \cos \varepsilon_{\nu}+c_{\mu} \operatorname{sen} \varepsilon_{\nu}\right) \\
\Sigma I_{i} \frac{\partial i_{\lambda}}{\partial \nu}=\operatorname{sen} \eta_{\nu} \cos \varepsilon_{\mu}\left(\beta_{\nu}+\frac{\partial \varepsilon_{\mu}}{\partial \nu}\right)-\cos \eta_{\nu}\left(\alpha_{\nu} \cos \varepsilon_{\mu}-\gamma_{\nu} \operatorname{sen} \varepsilon_{\mu}\right) .
\end{array}\right\}
$$

Le stesse (39) somministrano

$\Sigma I_{\nu} \frac{\partial i_{\mu}}{\partial \nu}=\frac{V \Delta}{\operatorname{sen} \varepsilon_{\nu}} \operatorname{sen} \varepsilon_{\lambda}\left(b_{\nu}-\frac{\partial \varepsilon_{2}}{\partial \nu}\right)$ 


$$
\begin{aligned}
& +\sum I_{\nu} i_{\nu}^{\prime}\left(-\frac{\partial \operatorname{sen} \varepsilon_{\lambda}}{\partial \nu} \operatorname{sen} \omega_{\nu}+n^{\prime} \cos \varepsilon_{\lambda}+c_{\nu} \operatorname{sen} \varepsilon_{\lambda} \cos \omega_{\nu}\right) \\
& +\sum I_{\nu} i^{\prime \prime}{ }_{\nu}\left(\frac{\partial \operatorname{sen} \varepsilon_{\lambda}}{\partial \nu} \cos \omega_{\nu}+c_{2} \operatorname{sen} \varepsilon_{\lambda} \operatorname{sen} \omega_{\nu}\right) \\
\Sigma I_{\mu} \frac{\partial i_{\nu}}{\partial \mu}=-\frac{V \Delta}{\operatorname{sen} \varepsilon_{\mu}} \operatorname{sen} \varepsilon_{\lambda}\left(\beta_{\mu}+\frac{\partial \varepsilon_{2}}{\partial \mu}\right) & \\
& +\sum I_{\mu} i_{\mu}^{\prime}\left(\frac{\partial \operatorname{sen} \varepsilon_{2}}{\partial \mu} \operatorname{sen} \omega_{\mu}^{\prime}+m^{\prime} \cos \varepsilon_{\lambda}-\gamma_{\mu} \operatorname{sen} \varepsilon_{\lambda} \cos \omega^{\prime}{ }_{\mu}\right) \\
& -\sum I_{\mu} i^{\prime \prime}{ }_{\mu}\left(\frac{\partial \operatorname{sen} \varepsilon_{2}}{\partial \mu} \cos \omega^{\prime}{ }_{\mu}+\gamma_{\mu} \operatorname{sen} \varepsilon_{\lambda} \operatorname{sen}^{\prime} \omega_{\mu}\right) .
\end{aligned}
$$

I valori de' coseni $\sum I_{\mu} i^{\prime}{ }_{\mu}, \sum I_{\nu} i^{\prime}{ }_{\nu}$ si trovano assoggettando alle due permutazioni semplice e doppia l'equazione in $\Sigma I_{2} \frac{\partial i_{2}}{\partial \lambda}$ numero precedente scritta sotto la forma

$$
\left.\begin{array}{rl}
\Sigma I_{\lambda} i^{\prime}{ }_{\lambda} & =-\cos \eta_{\mu} \cos \omega^{\prime},-\operatorname{sen} \eta_{\mu} \operatorname{sen} \omega_{\lambda}^{\prime} \cos \varepsilon_{\nu} \\
& =-\cos \eta_{\nu} \cos \omega_{\lambda}+\operatorname{sen} \eta_{\nu} \operatorname{sen} \omega_{\lambda} \cos \varepsilon_{\mu} .
\end{array}\right\}
$$

Si deduce dalla prima (37)

$\sum I_{\lambda} i_{\lambda}^{\prime \prime}{ }_{\lambda}=\cos \omega_{\mu}^{\prime}\left(\cos \omega_{\mu} \operatorname{sen} \omega^{\prime},-\operatorname{sen} \omega_{\mu} \cos \omega^{\prime}{ }_{\lambda} \cos \varepsilon_{\nu}\right)+$

$$
+\operatorname{sen} \omega_{\mu}^{\prime}\left(\operatorname{sen} \omega_{\mu} \operatorname{sen} \omega_{2}^{\prime}+\cos \omega_{\mu} \cos \omega_{2}^{\prime} \cos \varepsilon_{\nu}\right),
$$

ovvero

$$
\left.\begin{array}{rl}
\Sigma I_{\lambda} i^{\prime \prime}{ }_{2} & =-\cos \eta_{\mu} \operatorname{sen} \omega_{\lambda}^{\prime}+\operatorname{sen} \eta_{\mu} \cos \omega_{\lambda}^{\prime} \cos \varepsilon_{\nu} \\
& =-\cos \eta_{\nu} \operatorname{sen} \omega_{\lambda}-\operatorname{sen} \eta_{\nu} \cos \omega_{\lambda} \cos \varepsilon_{\mu} ;
\end{array}\right\}
$$

e da questa si concludono, mediante le due solite permutazioni, i valori de'coseni $\mathbf{\Sigma} I_{\mu} i^{\prime \prime}{ }_{\mu}, \mathbf{\Sigma} I_{\nu} i^{\prime \prime}{ }_{\nu}$. Avremo dunque, sostituendo i valori de' quattro coseni,

$$
\begin{aligned}
& \sum I_{\nu} \frac{\partial i_{\mu}}{\partial \nu}=\frac{V \Delta}{\operatorname{sen} \varepsilon_{\nu}} \operatorname{sen} \varepsilon_{\lambda}\left(b_{\nu}-\frac{\partial \varepsilon_{\lambda}}{\partial \nu}\right) \\
& -\left(\cos \eta_{\mu} \cos \omega_{\nu}-\operatorname{sen} \eta_{\mu} \operatorname{sen} \omega_{\nu} \cos \varepsilon_{\lambda}\right)\left(-\frac{\partial \operatorname{sen} \varepsilon_{\lambda}}{\partial \nu} \operatorname{sen} \omega_{\nu}+n^{\prime} \cos \varepsilon_{\lambda}+c_{\nu} \operatorname{sen} \varepsilon_{\lambda} \cos \omega_{\nu}\right) \\
& -\left(\cos \eta_{\mu} \operatorname{sen} \omega_{\nu}+\operatorname{sen} \eta_{\mu} \cos \omega_{\nu} \cos \varepsilon_{\lambda}\right)\left(\frac{\partial \operatorname{sen} \varepsilon_{2}}{\partial \nu} \cos \omega_{\nu}+c_{\nu} \operatorname{sen} \varepsilon_{\lambda} \operatorname{sen} \omega_{\nu}\right)
\end{aligned}
$$


$\sum I_{\mu} \frac{\partial i_{\nu}}{\partial \mu}=-\frac{V \Delta}{\operatorname{sen} \varepsilon_{\mu}} \operatorname{sen} \varepsilon_{2}\left(\beta_{\mu}+\frac{\partial \varepsilon_{2}}{\partial \mu}\right)$

$-\left(\cos \eta_{\nu} \cos \omega^{\prime} \mu+\operatorname{sen} \eta_{\nu} \operatorname{sen} \omega_{\mu}^{\prime} \cos \varepsilon_{2}\right)\left(\frac{\partial \operatorname{sen} \varepsilon_{\lambda}}{\partial \mu} \operatorname{sen} \omega_{\mu}^{\prime}+m^{\prime} \cos \varepsilon_{\lambda}-\gamma_{\mu} \operatorname{sen} \varepsilon_{2} \cos \omega^{\prime} \mu\right)$

$+\left(\cos \eta_{\nu} \operatorname{sen} \omega^{\prime} \mu-\operatorname{sen} \eta_{\nu} \cos \omega^{\prime} \mu \cos \varepsilon_{\eta}\right)\left(\frac{\partial \operatorname{sen} \varepsilon_{\lambda}}{\partial \mu} \cos \omega_{\mu}^{\prime}+\gamma_{\mu} \operatorname{sen} \varepsilon_{\lambda_{1}} \operatorname{sen} \omega_{\mu}^{\prime}\right)$

ovvero

$$
\begin{aligned}
\Sigma I_{\nu} \frac{\partial i_{\mu}}{\partial \nu} & =\operatorname{sen}^{2} \varepsilon_{\lambda} \operatorname{sen} \eta_{\mu}\left(b_{\nu}-\frac{\partial \varepsilon_{2}}{\partial \nu}\right)-\cos ^{2} \varepsilon_{\lambda} \operatorname{sen} \eta_{\mu} \frac{\partial \varepsilon_{\lambda}}{\partial \nu} \\
& -c_{\nu} \operatorname{sen} \varepsilon_{\lambda} \cos \eta_{\mu}-\cos \varepsilon_{\lambda}\left(a_{\nu} \cos \eta_{\mu}-b_{\nu} \operatorname{sen} \eta_{\mu} \cos \varepsilon_{\lambda}\right) \\
\Sigma I_{\mu} \frac{\partial i_{\nu}}{\partial \mu} & =-\operatorname{sen}^{2} \varepsilon_{\lambda} \operatorname{sen} \eta_{\nu}\left[\beta_{\mu}+\frac{\partial \varepsilon_{2}}{\partial \mu}\right)-\cos ^{2} \varepsilon_{\lambda} \operatorname{sen} \eta_{\nu} \frac{\partial \varepsilon_{\lambda}}{\partial \mu} \\
& +\gamma_{\mu} \operatorname{sen} \varepsilon_{\lambda} \cos \eta_{\nu}-\cos \varepsilon_{\lambda}\left(\alpha_{\mu} \cos \eta_{\nu}+\beta_{\mu} \operatorname{sen} \eta_{\nu} \cos \varepsilon_{2}\right)
\end{aligned}
$$

oppure

$$
\begin{aligned}
& \boldsymbol{\Sigma} I_{\nu} \frac{\partial i_{\mu}}{\partial \nu}=\operatorname{sen} \eta_{\mu}\left(b_{\nu}-\frac{\partial \varepsilon_{2}}{\partial \nu}\right)-\cos \eta_{\mu}\left(\alpha_{\nu} \cos \varepsilon_{\lambda}+c_{\nu} \operatorname{sen} \varepsilon_{2}\right), \\
& \Sigma I_{\mu} \frac{\partial i_{\nu}}{\partial \mu}=-\operatorname{sen} \eta_{\nu}\left(\beta_{\mu}+\frac{\partial \varepsilon_{\lambda}}{\partial \mu}\right)-\cos \eta_{\nu}\left(\alpha_{\mu} \cos \varepsilon_{\lambda}-\gamma_{\mu} \operatorname{sen} \varepsilon_{\lambda}\right) .
\end{aligned}
$$

Se ora prendiamo la permutazione doppia della prima di queste equazioni e la permutazione semplice della seconda, otteniamo

$$
\left.\begin{array}{l}
\boldsymbol{\Sigma} I_{\mu} \frac{\partial i_{\lambda}}{\partial \mu}=\operatorname{sen} \eta_{\lambda}\left(b_{\mu}-\frac{\partial \varepsilon_{\nu}}{\partial \mu}\right)-\cos \eta_{\lambda}\left(a_{\mu} \cos \varepsilon_{\nu}+c_{\mu} \operatorname{sen} \varepsilon_{\nu}\right), \\
\boldsymbol{\Sigma} I_{\nu} \frac{\partial i_{\lambda}}{\partial \nu}=-\operatorname{sen} \eta_{\lambda}\left(\beta_{\nu}+\frac{\partial \varepsilon_{\mu}}{\partial \nu}\right)-\cos \eta_{\lambda}\left(\alpha_{\nu} \cos \varepsilon_{\mu}-\gamma_{\nu} \operatorname{sen} \varepsilon_{\mu}\right) .
\end{array}\right\}
$$

Finalmente, se prendiamo la permutazione doppia della (40) e la permutazione semplice della (41), troviamo

$$
\begin{aligned}
& \mathbf{\Sigma} I_{\nu} \frac{\partial i_{\lambda}}{\partial \mu}=a_{\mu} \cos \varepsilon_{\nu}+c_{\mu} \operatorname{sen} \varepsilon_{\nu} \\
& \mathbf{\Sigma} I_{\mu} \frac{\partial i_{\lambda}}{\partial \nu}=\alpha_{\nu} \cos \varepsilon_{\mu}-\gamma_{\nu} \operatorname{sen} \varepsilon_{\mu} .
\end{aligned}
$$


3.

Cid posto, esprimiamo le diciotto componenti oblique della curvatura inclinata delle tre linee coordinate in funzioni delle $l, \gamma n, n, \varepsilon_{\lambda}, \varepsilon_{\mu}, \varepsilon_{\nu}$ e loro derivate prime.

La curvatura inclinata, cioè l'unità divisa pel raggio di curvatura inclinata, della linea $(\nu, \lambda)$. secondo la $(\mu, \nu)$ ha le tre projezioni sopra i tre assi rettilinei rappresentate da $\frac{1}{m} \frac{\partial i_{j}}{\partial \mu}$; e la curvatura inclinata della $(\lambda, \mu)$ secondo la stessa $(\mu, \nu)$ ha le tre projezioni rappresentate da $\frac{1}{n} \frac{\partial i_{\mu}}{\partial \nu}$. Chiamiamo $\frac{1}{L_{\lambda \mu}}, \frac{1}{M_{2 \mu}}, \frac{1}{N_{\lambda \mu}}$ le tre componenti oblique dell'una curvatura inclinata secondo le tangenti alle tre linee coordinate $(\mu, \nu),(\nu, \lambda),(\lambda, \mu)$, cioè le tre componenti formate dietro la regola del parallelepipedo delle forze; e chiamiamo $\frac{1}{L_{2 \nu}}, \frac{1}{M_{2 \nu}}, \frac{1}{N_{2 \nu}}$ le tre componenti oblique dell'altra curvatura inclinata. Gl'indici in basso di ciascuna lettera sono scritti in modo che il primo tra essi dinota sempre la linea a cui rimane tangente la retta di coseni sottoposti a variazione, ed il secondo la linea lungo cui la variazione è valutata. Sussisteranno le

$$
\left.\begin{array}{l}
\frac{1}{m} \frac{\partial i_{2}}{\partial \mu}=\frac{i_{\lambda}}{L_{\eta_{\mu}}}+\frac{i_{\mu}}{M_{\lambda_{\mu}}}+\frac{i_{\nu}}{N_{\lambda_{\mu}}}, \\
\frac{1}{n} \frac{\partial i_{2}}{\partial \nu}=\frac{i_{2}}{L_{\lambda_{\nu}}}+\frac{i_{\mu}}{M_{\lambda_{\nu}}}+\frac{i_{\nu}}{N_{\lambda_{\nu}}} \cdot
\end{array}\right\}
$$

Moltiplicando queste equazioni successivamente per $I_{\lambda}, I_{\mu}, I_{\nu}$ ed operando ad ogni volta la somma $\mathbf{y}$ sopra i prodotti, si trovano, in causa de'valori 
costituiti nel numero precedente,

$\frac{m \bigvee \Delta}{L_{\lambda, \mu}}=-\operatorname{sen} \varepsilon_{\lambda}\left\{\operatorname{sen} \eta_{\mu} \cos \varepsilon_{\nu}\left(b_{\mu}-\frac{\partial \varepsilon_{\nu}}{\partial \mu}\right)+\cos \eta_{\mu}\left(a_{\mu} \cos \varepsilon_{\nu}+c_{\mu} \operatorname{sen} \varepsilon_{\nu}\right)\right\}$,

$\frac{m V \Delta}{M_{\lambda \mu}}=\operatorname{sen} \varepsilon_{\mu}\left\{\operatorname{sen} \eta_{\lambda}\left(b_{\mu}-\frac{\partial \varepsilon_{\nu}}{\partial \mu}\right)-\cos \eta_{\lambda}\left(a_{i \iota} \cos \varepsilon_{\nu}+c_{\mu} \operatorname{sen} \varepsilon_{\nu}\right)\right\}$,

$\frac{m \bigvee \Delta}{N_{2, \mu}}=\operatorname{sen} \varepsilon_{\nu}\left(a_{\mu} \cos \varepsilon_{\nu}+c_{\mu} \operatorname{sen} \varepsilon_{\nu}\right)$

$\frac{n \bigvee \Delta}{L_{j, \nu}}=\operatorname{sen} \varepsilon_{\lambda}\left\{\operatorname{sen} \eta_{\nu} \cos \varepsilon_{\mu}\left(\beta_{\nu}+\frac{\partial \varepsilon_{\mu}}{\partial \nu}\right)-\cos \eta_{\nu}\left(\alpha_{\nu} \operatorname{coo} \varepsilon_{\mu}-\gamma_{\nu} \operatorname{sen} \varepsilon_{\mu}\right)\right\}$

$\frac{n \bigvee \Delta}{M_{\lambda_{\nu}}}=\operatorname{sen} \varepsilon_{\mu}\left(\alpha_{\nu} \cos \varepsilon_{\mu}-\gamma_{\nu} \operatorname{sen} \varepsilon_{\mu}\right)$,

$\frac{n V \Delta}{N_{\lambda, \nu}}=-\operatorname{sen} \varepsilon_{\nu}\left\{\operatorname{sen} \eta_{\lambda}\left(\beta_{\nu}+\frac{\partial \varepsilon_{\mu}}{\partial \nu}\right)+\cos \eta_{\lambda}\left(\alpha_{\nu} \cos \varepsilon_{\mu}-\gamma_{\nu} \operatorname{sen} \varepsilon_{\mu}\right)\right\}$

Queste equazioni esprimono le tre componenti oblique della curvatura inclinata della $(\nu, \lambda)$ secondo la $(\mu, \nu)$ e le tre della $(\lambda, \mu)$ secondo la stessa $(\mu, \nu)$ nel modo dichiarato, purchè s'intendano sostituiti in vece delle $b_{\mu}$, $a_{\mu}, \beta_{\nu}, \alpha_{\nu}$ i valori dati dalle (62), (63), (64) ed invece delle $c_{\mu}, \gamma_{2}$ i valori dati dalle (69) in cui siansi eliminati $i$ tre angoli $\theta$ col mezzo delle (70). Esse equazioni somministreranno con la permutazione semplice le tre componenti oblique della $(\lambda, \mu)$ e le tre della $(\mu, \nu)$ secondo la $(\nu, \lambda)$; e samministreranno con la permutazione doppia le tre componenti oblique della $(\mu, \nu)$ e le tre della $(\nu, \lambda)$ secondo la $(\lambda, \mu)$.

Se le superficie de' tre sistemi sono ortogonali tra loro, le (83) si riducono alle

$$
\begin{aligned}
& \frac{1}{L_{\lambda, \mu}}=0, \quad \frac{1}{M_{\lambda \mu}}=\frac{1}{\operatorname{lm}} \frac{\partial m}{\partial \lambda}, \quad \frac{1}{N_{\lambda \mu}}=0, \\
& \frac{1}{L_{\lambda \nu}}=0, \quad \frac{1}{M_{\lambda \nu}}=0, \quad \frac{1}{N_{\lambda \nu}}=\frac{1}{\ln } \frac{\partial n}{\partial \lambda} .
\end{aligned}
$$

4.

Osserviamo che, col sostituire nella (76) i valori dati dalle (77) in luogo delle tre componenti oblique della curvatura, e col sostituire nelle (82) i 
valori dati dalle (83) in luogo delle sei componenti oblique della curvatura inclinata, si troverebbero le tre variazioni del coseno $i$, lungo le tre linee coordinate espresse mediante le $l, m, n, \varepsilon_{2}, \varepsilon_{\mu}, \varepsilon_{\nu}$ e loro derivate prime, oltre le $i_{2}, i_{\mu}, i_{\nu}$. Indi, con le due permutazioni semplice e doppia, si dedurrebbero le tre variazioni del coseno $i_{\mu}$ e le tre del coseno $i_{\nu}$ espresse in modo analogo. Possiamo adunque risguardare queste nove variazioni indifferentemente come espresse nel modo or ora dichiarato, oppure come espresse mediante le componenti oblique, oltre le $i_{\lambda}, i_{\mu}, i_{\nu}$, in causa delle (76), (82). Ora, intorno alle due forme sotto alle quali i valori di queste nove variazioni possono essere presentati, il sig. Aoust cosi si esprime alla pag. 80 degli Annali citati: Ces variations constituent en quelque sorte l'essence de la question qui nous occupe. Dans la théorie purement analytique des coordonnées curvilignes, elles dépendent de la résolution d'un système de 9 équations linéaires à 9 inconnues, dont les coefficients se composent des variations premières et secondes des paramètres différentiels du premier ordre et des variations des angles coordonnés. La méthode que nous avons suivie, dispense de la résolution d'un pareil système d'équations, résolution qui serait peut-être impraticable; elle y substitue un procédé simple et facile, et donne l'expression de ces variations sous une forme à laquelle l'introduction des courbures inclinées donne un caractère de clarté et de symétrie.

5.

Passiamo ora a formare le equazioni finite, che legano tra loro le nove componenti oblique della curvatura, le diciotto componenti oblique della curvatura inclinata ed i tre angoli coordinati: equazioni già costituite con altro metodo dal sig. AousT.

I.a (76), moltiplicata per $i$, e sottoposta all'operazione $\mathbf{\Sigma}$, dà

$$
\frac{1}{L_{2}}+\frac{\cos \varepsilon_{\nu}}{M_{2}}+\frac{\cos \varepsilon_{\mu}}{N_{2}}=0
$$

dalla quale si deducono altre due mediante le due solite permutazioni. Cosi, 
le due (82), moltiplicate per $i_{\lambda}$ e sottoposte alla stessa somma $\mathbf{\Sigma}$, danno

$$
\left.\begin{array}{c}
\frac{1}{L_{2, \mu}}+\frac{\cos \varepsilon_{\nu}}{M_{2 \mu}}+\frac{\cos \varepsilon_{\mu}}{N_{2 \mu}}=0, \\
\frac{1}{L_{2, \nu}}+\frac{\cos \varepsilon_{\nu}}{M_{2 \nu}}+\frac{\cos \varepsilon_{\mu}}{N_{2,}}=0 ;
\end{array}\right\}
$$

da ciascuna delle quali si deducono altre due col mezzo delle stesse due permutazioni. La (84) e la seconda (85) sono le relazioni registrate alla pag. 81 degli Annali del 1864.

Il confronto fra la permutazione semplice della terza (83) e la permutazione doppia della quinta, avuto riguardo alla (42), somministra

$$
\frac{1}{L_{\mu \nu}}=\frac{1}{L_{\nu \mu}}
$$

dalla quale si deducono altre due mediante le due solite permutazioni. La (86) è la relazione registrata alla pag. 86 de' medesimi Annali.

Abbiamo dunque dodici relazioni finite fra le trenta indicate quantità; cioè tre fra le nove componenti oblique della curvatura ed i tre angoli, e nove fra le diciotto componenti della curvatura inclinata e gli stessi tre angoli.

6.

Formeremo, partendo dalle (82), i valori delle $\frac{\partial l}{\partial \mu}, \frac{\partial l}{\partial \nu}, \frac{\partial m}{\partial \nu}, \frac{\partial m}{\partial \lambda}, \frac{\partial n}{\partial \lambda}, \frac{\partial n}{\partial \mu}$ in funzioni di dodici tra le diciotto componenti oblique della curvatura inclinata e delle $l, m, n$; valori già trovati con altro metodo dal sig. Aoust.

Moltiplichiamo la prima (82) per $i_{\mu}$, la seconda per $i_{\nu}$, e prendiamo sopra i due prodotti la somma $\mathbf{Z}$. In causa della seconda (13) e della prima (15), risulteranno

$$
\begin{aligned}
& \frac{1}{\operatorname{lm}}\left(\frac{\partial m}{\partial \lambda}-\frac{\partial l}{\partial \mu} \cos \varepsilon_{\nu}\right)=\frac{\cos \varepsilon_{\nu}}{L_{\lambda \mu}}+\frac{1}{M_{\lambda, \mu}}+\frac{\cos \varepsilon_{2}}{N_{\lambda \mu}} \\
& \frac{1}{n l}\left(\frac{\partial n}{\partial \lambda}-\frac{\partial l}{\partial \nu} \cos \varepsilon_{\mu}\right)=\frac{\cos \varepsilon_{\mu}}{L_{\lambda_{\nu}}}+\frac{\cos \varepsilon_{2}}{M_{\lambda_{\nu}}}+\frac{1}{N_{\eta_{\nu}}}
\end{aligned}
$$


Sommo la prima di queste equazioni moltiplicata per $\cos \varepsilon_{\nu} \operatorname{con}$ la permutazione semplice della seconda, ed ho

$$
\begin{gathered}
\frac{\operatorname{sen}^{2} \varepsilon_{\nu}}{l m} \frac{\partial l}{\partial \mu}=\frac{1-\operatorname{sen}^{2} \varepsilon_{\nu}}{L_{\mu}}+\frac{\cos \varepsilon_{\nu}}{M_{2 \mu}}+\frac{\cos \varepsilon_{\mu}-\operatorname{sen} \varepsilon_{\nu} \operatorname{sen} \varepsilon_{\lambda} \cos \eta_{\mu}}{N_{2 \mu}} \\
+\frac{1}{L_{\mu l}}+\frac{\cos \varepsilon_{\nu}}{M_{\mu l}}+\frac{\cos \varepsilon_{\mu}}{N_{\mu l}} ;
\end{gathered}
$$

ovvero, in causa della prima (85) e della permutazione doppia della (86),

$$
\frac{\operatorname{sen}^{2} \varepsilon_{\nu}}{l m} \frac{\partial l}{\partial \mu}=-\frac{\operatorname{sen}^{2} \varepsilon_{\nu}}{L_{\lambda \mu}}+\frac{1}{L_{\mu l}}+\frac{\cos \varepsilon_{\nu}}{M_{\mu l}}+\frac{\cos \varepsilon_{\nu} \cos \varepsilon_{2}}{N_{\mu z}}
$$

oppure, in causa della permutazione semplice della seconda (85),

$$
\frac{\partial l}{\partial \mu}=\ln \left(\frac{1}{L_{\mu 2}}-\frac{1}{L_{\lambda, \mu}}\right)
$$

dalla quale, mediante le due solite permutazioni, si concluderanno i valori delle $\frac{\partial m}{\partial \nu}, \frac{\partial n}{\partial \lambda}$. Dipoi, sommo la prima delle precedenti equazioni con la permutazione semplice della seconda moltiplicata per $\cos \varepsilon_{\nu}$, ed ho

$$
\begin{gathered}
\frac{\operatorname{sen}^{2} \varepsilon_{\nu}}{\operatorname{lm} \frac{\partial m}{\partial \lambda}=\frac{\cos \varepsilon_{\nu}}{L_{\mu \lambda}}}+\frac{1-\operatorname{sen}^{2} \varepsilon_{\nu}}{M_{\mu 2}}+\frac{\cos \varepsilon_{2}-\operatorname{sen} \varepsilon_{\mu} \operatorname{sen} \varepsilon_{\nu} \cos \eta_{2}}{N_{\mu \lambda}} \\
+\frac{\cos \varepsilon_{\nu}}{L_{\lambda, \mu}}+\frac{1}{M_{\lambda \mu}}+\frac{\cos \varepsilon_{2}}{N_{2, \mu}}
\end{gathered}
$$

ovvero, in causa della permutazione semplice della seconda (85) e della permutazione doppia della (86)

$$
\frac{\operatorname{sen}^{2} \varepsilon_{\nu}}{l m} \frac{\partial m}{\partial \lambda}=-\frac{\operatorname{sen}^{2} \varepsilon_{\nu}}{M_{\mu \lambda}}+\frac{\cos \varepsilon_{\nu}}{L_{\lambda_{\mu}}}+\frac{1}{M_{\lambda, \mu}}+\frac{\cos \varepsilon_{\mu} \cos \varepsilon_{\nu}}{N_{\lambda \mu}}
$$

oppure, in causa della prima (85),

$$
\frac{\partial m}{\partial \lambda}=\operatorname{lm}\left(\frac{1}{M_{\lambda \mu}}-\frac{1}{M_{\mu 2}}\right)
$$

dalla quale, mediante le due solite permutazioni, si concluderanno $i$ valori delle $\frac{\partial n}{\partial \mu}, \frac{\partial l}{\partial \nu}$. Le (87), (88) sono le relazioni registrate alla pag. 85 degli Annali citati. 
7.

Formiamo ora i valori delle $\frac{\partial \cos \varepsilon_{2}}{\partial \lambda}, \frac{\partial \cos \varepsilon_{\mu}}{\partial \mu}, \frac{\partial \cos \varepsilon_{\nu}}{\partial \nu}$ in funzioni delle componenti oblique della curvatura inclinata e delle $l, m, n, \varepsilon_{\eta}, \varepsilon_{\mu}, \varepsilon_{\nu}$.

Moltiplichiamo la prima (82) per $i_{\nu}$, e facciamo sul prodotto l'operazione ¿. In causa della seconda (16), risulterà

$\frac{1}{2 l m n}\left(\frac{\partial \cdot m n \cos \varepsilon_{2}}{\partial \lambda}-\frac{\partial \cdot l m \cos \varepsilon_{\nu}}{\partial \nu^{\prime}}+\frac{\partial \cdot n l \cos \varepsilon_{\mu}}{\partial \mu}-2 n \frac{\partial l}{\partial \mu} \cos \varepsilon_{\mu}\right)=$

$$
\frac{\cos \varepsilon_{\mu}}{L_{i \mu}}+\frac{\cos \varepsilon_{2}}{M_{i \mu}}+\frac{1}{N_{2 \mu}}
$$

ovvero, avuto riguardo alla (87),

$\frac{1}{2 \ln n}\left(\frac{\partial \cdot m n \cos \varepsilon_{2}}{\partial \lambda}-\frac{\partial \cdot \ln \cos \varepsilon_{2}}{\partial \nu}+\frac{\partial \cdot n l \cos \varepsilon_{\mu}}{\partial \mu}\right)=\frac{\cos \varepsilon_{\mu}}{L_{\mu \lambda}}+\frac{\cos \varepsilon_{2}}{M_{\lambda, \mu}}+\frac{1}{N_{\lambda \mu}}$

Cosi, moltiplichiamo la seconda (82) per $i_{\mu}$, e facciamo sul prodotto l'operazione $\mathbf{\Sigma}$. In causa della terza $(16)$, scrivendo

$$
\frac{1}{l m}\left(\frac{\partial \cdot \operatorname{lm} \cos \varepsilon_{\nu}}{\partial \nu}-\cos \varepsilon_{\nu} \frac{\partial \cdot l m}{\partial \nu}\right)-\Sigma i_{\lambda} \frac{\partial i_{\mu}}{\partial \nu}
$$

al posto di

$$
\mathbf{\Sigma} i_{\mu} \frac{\partial i_{i}}{\partial \nu}
$$

risulterà

$$
\begin{aligned}
\frac{1}{\operatorname{lm} n}\left(\frac{\partial \cdot l m \cos \varepsilon_{\nu}}{\partial \nu}-\cos \varepsilon_{\nu} \frac{\partial \cdot l m}{\partial \nu}\right)-\frac{1}{2 l m n}\left(\frac{\partial \cdot n l \cos \varepsilon_{\mu}}{\partial \mu}-\frac{\partial \cdot m n \cos \varepsilon_{2}}{\partial \lambda}\right. & \\
+ & \left.\frac{\partial \cdot l m \cos \varepsilon_{\nu}}{\partial \nu}-2 l \frac{\partial m}{\partial \nu} \cos \varepsilon_{\nu}\right)=\frac{\cos \varepsilon_{\nu}}{L_{\lambda_{\nu}}}+\frac{1}{M_{\lambda \nu}}+\frac{\cos \varepsilon_{\lambda}}{N_{\lambda \nu}}
\end{aligned}
$$

ovvero, avuto riguardo alla permutazione doppia della (88),

$\frac{1}{2 l m n}\left(\frac{\partial \cdot m n \cos \varepsilon_{2}}{\partial \lambda}+\frac{\partial \cdot l m \cos \varepsilon_{\nu}}{\partial \nu}-\frac{\partial \cdot n l \cos \varepsilon_{\mu}}{\partial \mu}\right)=\frac{\cos \varepsilon_{\nu}}{L_{\nu}}+\frac{1}{M_{\lambda, \nu}}+\frac{\cos \varepsilon_{\lambda}}{M_{\lambda,}} \cdot(b)$

Ora, sommando le $(a),(b)$, si trova

$$
\frac{1}{\operatorname{lm} n} \frac{\partial \cdot m n \cos \varepsilon_{2}}{\partial \lambda}=\frac{\cos \varepsilon_{\mu}}{L_{\mu \lambda}}+\frac{\cos \varepsilon_{2}}{M_{\lambda, \mu}}+\frac{1}{N_{\lambda, \mu}}+\frac{\cos \varepsilon_{\nu}}{L_{\nu \lambda}}+\frac{1}{M_{\lambda, \nu}}+\frac{\cos \varepsilon_{\lambda}}{N_{\lambda \nu}}
$$


ovvero, in causa della (88), della permutazione doppia della (87) e delle permutazioni semplice e doppia della $(86)$,

$$
\frac{1}{l} \frac{\partial \cos \varepsilon_{\lambda}}{\partial \lambda}=\frac{\cos \varepsilon_{\mu}}{L_{\mu \lambda}}+\frac{\cos \varepsilon_{\lambda}}{M_{\mu \lambda}}+\frac{1}{N_{\mu \lambda}}+\frac{\cos \varepsilon_{\nu}}{L_{\nu \lambda}}+\frac{1}{M_{\nu \lambda}}+\frac{\cos \varepsilon_{\lambda}}{N_{\nu \lambda}} ;
$$

dalla quale si deducono i valori delle $\frac{\partial \cos \varepsilon_{\mu}}{\partial \mu}, \frac{\partial \cos \varepsilon_{\nu}}{\partial \nu}$ mediante le due solite permutazioni. La (89) e quelle che ne conseguono esprimono le tre derivate parziali nel modo dichiarato. Nella (89) può trasformarsi l'equazione esprimente il valore della $\frac{\partial \varepsilon_{\lambda}}{\partial \lambda}$ registrata alla pag. 75 degli Annali suddetti.

\section{8.}

Da ultimo formiamo $i$ valori delle $\frac{\partial \cos \varepsilon_{9}}{\partial \mu}, \frac{\partial \cos \varepsilon_{\lambda}}{\partial \nu}, \frac{\partial \cos \varepsilon_{\mu}}{\partial \nu}, \frac{\partial \cos \varepsilon_{\mu}}{\partial \lambda}$, $\frac{\partial \cos \varepsilon_{\nu}}{\partial \lambda}, \frac{\partial \cos \varepsilon_{\nu}}{\partial \mu}$ in funzioni delle componenti oblique delle due curvature ordinaria ed inclinata e delle $l, m, n, \varepsilon_{2}, \varepsilon_{\mu}, \varepsilon_{\nu}$.

Moltiplichiamo la (76) per $i_{\mu}$ e prendiamo sul prodotto la somma $\mathbf{\Sigma}$. In causa della prima (13) avremo

$$
\frac{1}{l} \frac{\partial \cos \varepsilon_{\nu}}{\partial \lambda}-\frac{1}{l m}\left(\frac{\partial l}{\partial \mu}-\frac{\partial m}{\partial \lambda} \cos \varepsilon_{\nu}\right)=\frac{\cos \varepsilon_{\nu}}{L_{2}}+\frac{1}{M_{\lambda}}+\frac{\cos \varepsilon_{2}}{N_{2}}
$$

ovvero, avendo riguardo alle (87), (88),

$$
\frac{1}{l} \frac{\partial \cos \varepsilon_{\nu}}{\partial \lambda}=\frac{1}{L_{\mu \lambda}}+\frac{\cos \varepsilon_{\nu}}{M_{\mu \lambda}}-\frac{1}{L_{\lambda, \mu}}-\frac{\cos \varepsilon_{\nu}}{M_{\lambda, \mu}}+\frac{\cos \varepsilon_{\nu}}{L_{\lambda}}+\frac{1}{M_{\lambda}}+\frac{\cos \varepsilon_{\lambda}}{N,} ;
$$

0 anche, dietro la prima (85) la permutazione doppia della (86),

$$
\frac{1}{l} \frac{\partial \cos \varepsilon_{\nu}}{\partial \lambda}=\frac{1}{L_{\mu \lambda}}+\frac{\cos \varepsilon_{\nu}}{M_{\mu \lambda}}+\frac{\cos \varepsilon_{\mu}}{N_{\mu \lambda}}+\frac{\cos \varepsilon_{\nu}}{L_{\lambda}}+\frac{1}{M_{\lambda}}+\frac{\cos \varepsilon_{\lambda}}{N_{\lambda}} ;
$$

dalla quale si deducono altre due mediante le due solite permutazioni. Dipoi, moltiplichiamo la (76) per $i_{\nu}$ e prendiama ancora sul prodotto la somma $\mathbf{2}$; in causa della seconda (15) avremo

$$
\frac{1}{l} \frac{\partial \cos \varepsilon_{\mu}}{\partial \lambda}-\frac{1}{n l}\left(\frac{\partial l}{\partial \nu}-\frac{\partial n}{\partial \lambda} \cos \varepsilon_{\mu}\right)=\frac{\cos \varepsilon_{\mu}}{L_{\lambda}}+\frac{\cos \varepsilon_{\lambda}}{M_{\lambda}}+\frac{1}{N_{\lambda}}
$$


ovvero, avuto riguardo alle permutazioni doppie delle (87), (88),

$$
\frac{1}{l} \frac{\partial \cos \varepsilon_{\mu}}{\partial \lambda}=\frac{1}{L_{\nu \lambda}}+\frac{\cos \varepsilon_{\mu}}{N_{\nu \lambda}}-\frac{1}{L_{\gamma_{\nu}}}-\frac{\cos \varepsilon_{\mu}}{N_{2 \nu}}+\frac{\cos \varepsilon_{\mu}}{L_{2}}+\frac{\cos \varepsilon_{\lambda}}{M,}+\frac{1}{N_{2}}
$$

- anche, dietro la seconda (85) e la permutazione semplice della (86),

$$
\frac{1}{l} \frac{\partial \cos \varepsilon_{\mu}}{\partial \lambda}=\frac{1}{L_{\nu \lambda}}+\frac{\cos \varepsilon_{\nu}}{M_{\nu \lambda}}+\frac{\cos \varepsilon_{\mu}}{N_{\nu \lambda}}+\frac{\cos \varepsilon_{\mu}}{L_{\lambda}}+\frac{\cos \varepsilon_{2}}{M_{\lambda}}+\frac{1}{N_{\lambda}}
$$

dalla quale pure si deducono altre due mediante le due solite permutazioni. Le $(90)$ e (91) con quelle che ne conseguono esprimono le sei derivate parziali nel modo dichiarato. Nella (90) può trasformarsi l'equazione esprimente il valore della $\frac{\partial \varepsilon_{\lambda}}{\partial \mu}$ registrata alla pag. 75 degli Annali suddetti.

\section{Osservazione.}

Il sig. Aoust costituisce anche le equazioni contenenti le derivate parziali delle ventisette componenti oblique. A tal fine egli deduce dalle (76), (82) due diverse espressioni per ciascuna delle derivate seconde $\frac{\partial^{2} i_{2}}{\partial \lambda \partial \mu}, \frac{\partial^{2} i_{\lambda}}{\partial \mu \partial \nu}$, le eguaglia tra loro e rende identiche le equazioni risultanti. Noi non passeremo a formare queste equazioni.

Pavia, 18 luglio 1869. 